Supporting Information for:

\title{
Diphenylnitrenium Ion: Cyclization, Electron Transfer, and Polymerization Reactions
}

\author{
Andrew C. Kung, Sean P. McIlroy, and Daniel E. Falvey* \\ Department of Chemistry and Biochemistry, University of Maryland, College Park, MD \\ 20742-2021.
}

1. Photolysis of 2 with $N, N$-Diphenylamine (DPA). $\quad \mathrm{S} 2$

2. Photolysis of 2 with Carbazole. $\quad$ S2

3. Photolysis of 2 with $N, N$-Dimethylaniline (DMA). $\quad \mathrm{S} 4$

4. ${ }^{1} \mathrm{H}$ NMR Spectrum of $\mathbf{1 5 .}$ S7.

5. ${ }^{1} \mathrm{H}$ NMR Spectrum of $\mathbf{1 4} \quad$ S8

6. ${ }^{13} \mathrm{C}$ NMR Spectrum of $\mathbf{1 4} \quad$ S9

7. ${ }^{1} \mathrm{H}$ NMR Spectrum of $\mathbf{1 3} \quad$ S10

8. ${ }^{13} \mathrm{C}$ NMR Spectrum of $\mathbf{1 3} \quad \mathrm{S} 11$

9. ${ }^{13}$ C DEPT Spectrum of $\mathbf{1 3} \quad \mathrm{S} 12$

10. DFT Geometries for 10, 11, 12 
Photolysis of 2 with $N, N$-Diphenylamine (DPA). Several photolysis were performed using room light. $25 \mathrm{~mL}$ solutions of $0.0324 \mathrm{~g}(0.086 \mathrm{mmol}) \mathrm{N}, N$-diphenylaminyl-2,4,6trimethylpyridinium tetrafluoroborate and $0.3304 \mathrm{~g}(2.0 \mathrm{mmol}) \mathrm{DPA}$ was purged for 10 min with $\mathrm{N}_{2}$ then sealed with a rubber septum. The solution was placed under room light for 2 days during which the solution became clear. Removal of solvent in vacuo yielded a light yellow oil. The oil was taken up in a minimum amount of $\mathrm{Et}_{2} \mathrm{O}$ and directly added to a flash column $(45 \mathrm{~cm} \mathrm{x} 4 \mathrm{~cm})$ that had been packed with $10 \mathrm{~cm}$ of silica gel, 40 mesh. The column was eluted with a gradual increase in polarity starting from $500 \mathrm{~mL}$ hexanes then $500 \mathrm{~mL} 2.5 \% \mathrm{Et}_{2} \mathrm{O}$ :hexanes then $10 \% \mathrm{Et}_{2} \mathrm{O}$ :hexanes and finally $1000 \mathrm{~mL} 15 \%$ $\mathrm{Et}_{2} \mathrm{O}$ :hexanes and fractions were collected which were analyzed by GC. Combining appropriate fractions and concentrating in vacuo, the following photoproducts were obtained: $N, N, N$ '-triphenyl-1,4-phenylenediamine (14) ${ }^{1} \mathrm{H}$ NMR (400 MHz; $\left.\mathrm{CDCl}_{3}\right)$ : $\delta$ 7.26-7.21 (m, 6H), 7.08-6.94 (m, 12H), 6.87-6.83 (m, 1H), 6.64 (br s, 1H); ${ }^{13} \mathrm{C} \mathrm{NMR}$ $\left(110 \mathrm{MHz}, \mathrm{CDCl}_{3}\right) \delta 148.0,143.6,141.4,138.8,129.3,129.1,126.6,123.1,122.0,120.6$ 119.5, 117.2.

Photolysis of 2 with Carbazole. In a $50 \mathrm{~mL}$ Erlenmeyer flask wrapped in aluminum foil, $20 \mathrm{~mL}$ of freshly distilled $\mathrm{MeCN}$ was added to $0.0595 \mathrm{~g}(0.158 \mathrm{mmol})$ of $\mathrm{N}, \mathrm{N}$ diphenylaminyl-2,4,6-trimethylpyridinium tetrafluoroborate. To this solution, $0.0413 \mathrm{~g}$ $(0.247 \mathrm{mmol})$ of carbazole was added and the Erlenmeyer flask was capped with a rubber septum. The light yellow solution was purged under $\mathrm{N}_{2}$ for 10 min., unwrapped, and then allowed to sit under room light for $48 \mathrm{~h}$. After the $48 \mathrm{~h}$ period, the solution was charcoal in color and the solvent was removed in vacuo. 
A Shimadzu Corporation GC-17a equipped with a $15 \mathrm{~m} 0.25 \mathrm{~mm}$ I.D. 0.25 _m film DB-5 column was used. All injections were $1 \_\mathrm{L}$, and the temperature/pressure program was, $60^{\circ} \mathrm{C}$ at $67 \mathrm{kPa}$ for $3 \mathrm{~min}$ ramp at a rate of $30^{\circ} \mathrm{C}$ and $3.8 \mathrm{kPa}$ per minute finishing at $300^{\circ} \mathrm{C}$ and $97 \mathrm{kPa}$ which was held for 9 minutes. The column flow rate was $1.9 \mathrm{~mL} / \mathrm{min}$. The compounds were detected using a flame ionization detector. The GC trace of the photolysate showed four major peaks: one peak corresponding to diphenyl amine, one corresponding to carbazole, and two other products.

The remaining solid was taken up in a minimal amount of dichloromethane and added to a flash column $(45 \mathrm{~cm} \times 4 \mathrm{~cm})$ that had been packed with $36 \mathrm{~cm}$ of silica gel, 40 mesh. The column was eluted with a gradual increase in polarity starting from $500 \mathrm{~mL}$ hexanes, then $1 \mathrm{~L} 2 \% \mathrm{Et}_{2} \mathrm{O} /$ hexanes, then $1 \mathrm{~L} 10 \% \mathrm{Et}_{2} \mathrm{O} /$ hexanes, then $1 \mathrm{~L} 20 \% \mathrm{Et}_{2} \mathrm{O} /$ hexanes, then $1 \mathrm{~L} 40 \% \mathrm{Et}_{2} \mathrm{O} /$ hexanes. The collected fractions were analyzed by GC. The appropriate fractions were combined and concentrated under reduced pressure. The photoproduct was determined to be (4-carbazol-9-yl-phenyl)-phenyl-amine $\mathbf{1 3}$ by MS, ${ }^{1} \mathrm{H}$ NMR, ${ }^{13} \mathrm{C}$ NMR, and ${ }^{13} \mathrm{C}$ DEPT NMR and yielded $0.0141 \mathrm{~g}(0.042 \mathrm{mmol})$ of product. 1H NMR (400 MHz; CD3CN): _ 8.18 (d, 2H), $7.42(\mathrm{t}, 4 \mathrm{H}), 7.36$ (d, 2H), 7.32-7.25 (m, 5H), $7.21(\mathrm{~d}, 2 \mathrm{H}), 6.97(\mathrm{t}, 2 \mathrm{H}) ;{ }^{13} \mathrm{C} \mathrm{NMR}\left(400 \mathrm{MHz} ; \mathrm{CD}_{3} \mathrm{Cl}\right)$ : $_{-}$142.8, 142.4, 141.3, $130.0,129.5,128.3,125.8,123.0,121.8,120.2,119.6,118.6,117.9,109.8 ;{ }^{13} \mathrm{C}$ DEPT NMR (400 MHz; $\left.\mathrm{CD}_{3} \mathrm{Cl}\right)$ : $129.5,128.3,125.8,121.8,120.2,119.6,118.6,117.9$, 109.7; MS (FAB) m/z 334.5, 259.4, 241.4, 169.3, 168.3; HRMS (FAB) calcd for $\mathrm{C}_{24} \mathrm{H}_{18} \mathrm{~N}_{2} 334.1470$ found 334.1479

Concentration curves of diphenyl amine, carbazole, and the photoproduct were created via GC and used to determine the yield of the photolysis. These curves were generated 
from solutions with concentrations ranging from $1.97 \times 10^{-3} \mathrm{M}$ to $1.84 \times 10^{-1} \mathrm{M}$. The samples were analyzed via GC with the area underneath the curve for the compound recorded. The data were fit using a least-squares computer routine to obtain a straight line, $y=m x+b$, where $y$ is the peak area and $x$ is the concentration.

To determine that percent yield upon photolysis, another trial was performed with 0.0607 $\mathrm{g}(0.1618 \mathrm{mmol})$ of $N, N$-diphenylaminyl-2,4,6-trimethylpyridinium tetrafluoroborate in a $50 \mathrm{~mL}$ Erlenmeyer flask wrapped in aluminum foil and $20 \mathrm{~mL}$ of freshly distilled $\mathrm{MeCN}$. To this solution, $0.0455 \mathrm{~g}(0.272 \mathrm{mmol})$ of carbazole was added and the Erlenmeyer flask was capped with a rubber septum. The light yellow solution was purged under $\mathrm{N}_{2}$ for 10 min., unwrapped, and then allowed to sit under room light for $48 \mathrm{~h}$. At this time the solution had changed to charcoal in color and $2 \mathrm{~mL}$ was removed for analysis. The solution was kept under room light for an additional $96 \mathrm{~h}$. After the total of $144 \mathrm{~h}$, another sample was analyzed by GC. Assuming 100\% photolytic conversion and using the previously calculated concentration curves, the percent of photoadduct observed was $24 \%$ and the percent of dipheylamine observed was $51 \%$.

Photolysis of 2 with $N, N$-Dimethylaniline (DMA). $\quad$ Several photolysis were performed using room light. $25 \mathrm{~mL}$ solutions of $0.0342 \mathrm{~g}(0.091 \mathrm{mmol}) \mathrm{N}, \mathrm{N}-$ diphenylaminyl-2,4,6-trimethylpyridinium tetrafluoroborate and $300 \mu \mathrm{L}(2.1 \mathrm{mmol})$ DMA was purged for 10 min with $\mathrm{N}_{2}$ then sealed with a rubber septum. The solution was placed under room light for 2 days during which the solution became clear. Removal of solvent in vacuo yielded a light yellow oil. The oil was taken up in a minimum amount of $\mathrm{Et}_{2} \mathrm{O}$ and directly added to a flash column $(45 \mathrm{~cm} \mathrm{x} 4 \mathrm{~cm})$ that had been packed with 
$10 \mathrm{~cm}$ of silica gel, 40 mesh. The column was eluted with a gradual increase in polarity starting from $500 \mathrm{~mL}$ hexanes then $500 \mathrm{~mL} 2.5 \% \mathrm{Et}_{2} \mathrm{O}$ :hexanes then $10 \% \mathrm{Et}_{2} \mathrm{O}$ :hexanes

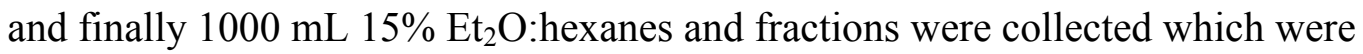
analyzed by GC. Combining appropriate fractions and concentrating in vacuo, the following photoproducts were obtained: $N, N$-dimethyl- $N$ ', $N$ '-diphenyl-1,4phenylenediamine (15) ${ }^{1} \mathrm{H}$ NMR $\left(400 \mathrm{MHz} ; \mathrm{CDCl}_{3}\right): \delta$ 7.19-7.15 (t, $\left.8 \mathrm{~Hz}, 4 \mathrm{H}\right), 7.03-7.01$ (d, $8 \mathrm{~Hz}, 4 \mathrm{H}), 6.91-6.87(\mathrm{t}, 8 \mathrm{~Hz}, 1 \mathrm{H}), 6.69-6.67$ (d, $8 \mathrm{~Hz}, 2 \mathrm{H}) 2.92(\mathrm{~s}, 6 \mathrm{H}) ;{ }^{13} \mathrm{C} \mathrm{NMR}$ $\left(110 \mathrm{MHz}, \mathrm{CDCl}_{3}\right) \delta$ 148.3, 147.7, 137.1, 128.9, 127.7, 122.4, 121.3, 113.6, 40.9.

\section{Product distributions with Varying Carbazole Concentrations.}

Compound 2 (2-4 mg) was dissolved in $20 \mathrm{~mL}$ dry $\mathrm{CH} 3 \mathrm{CN}$. To these solutions were added 2- $16 \mathrm{mg}$ of carbazole. The solutions were purged with $\mathrm{N} 2$ and then exposed to ambient light for $48 \mathrm{~h}$. The resulting solutions were analyzed by gas chromatography as described above. 


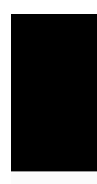

8

8हโ' 1

2

光

$599^{\circ} 9$
$489^{\circ}$
57

[ $\left.48^{\circ} \cdot 9\right]$

$688^{\circ} 9$

$=$

206.9

$210 . \mathrm{L}=$

5SI L-

DLT: $L-$

E6 ' $L$

$0 D 2^{\circ} L$

ưdd

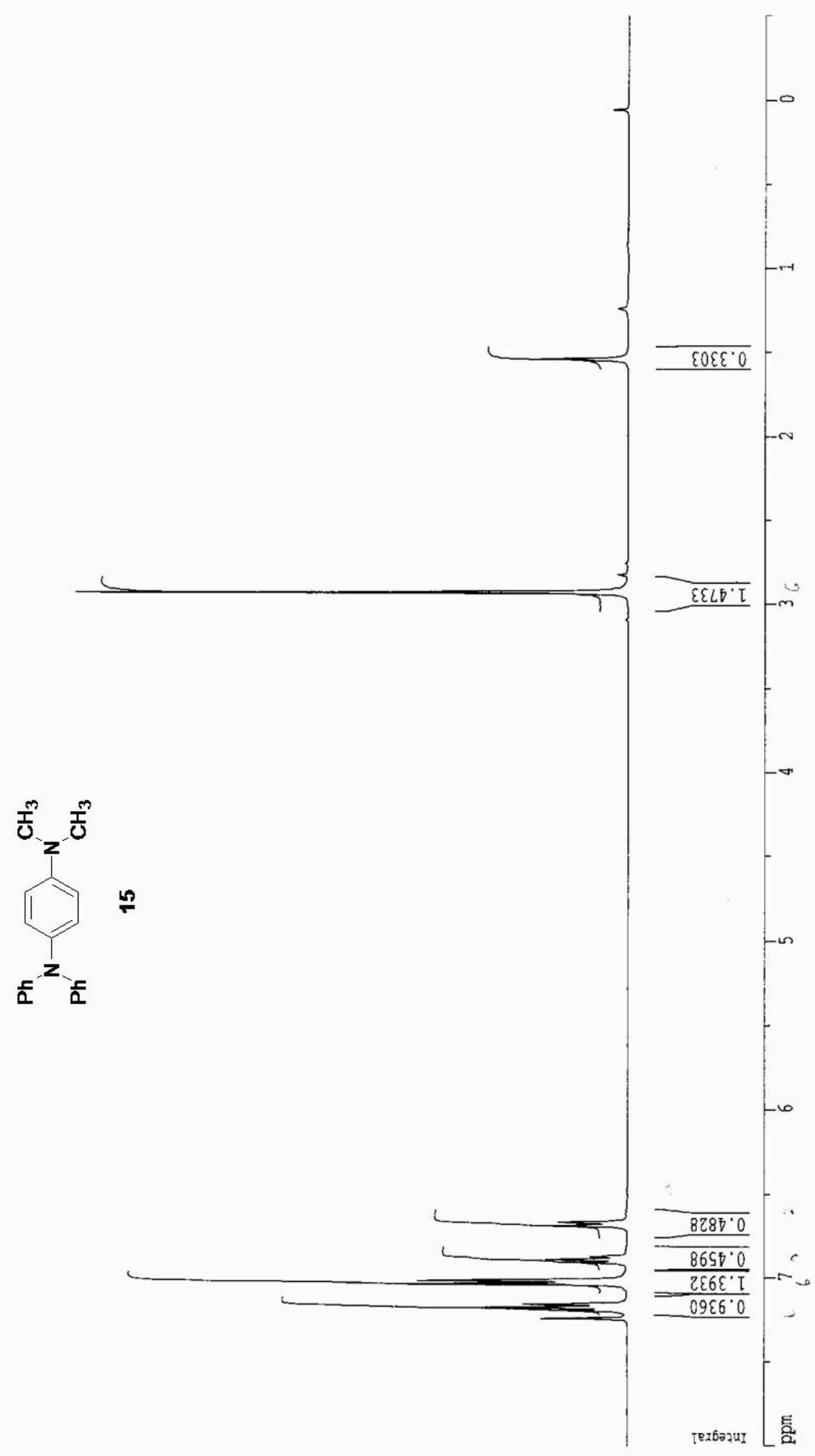




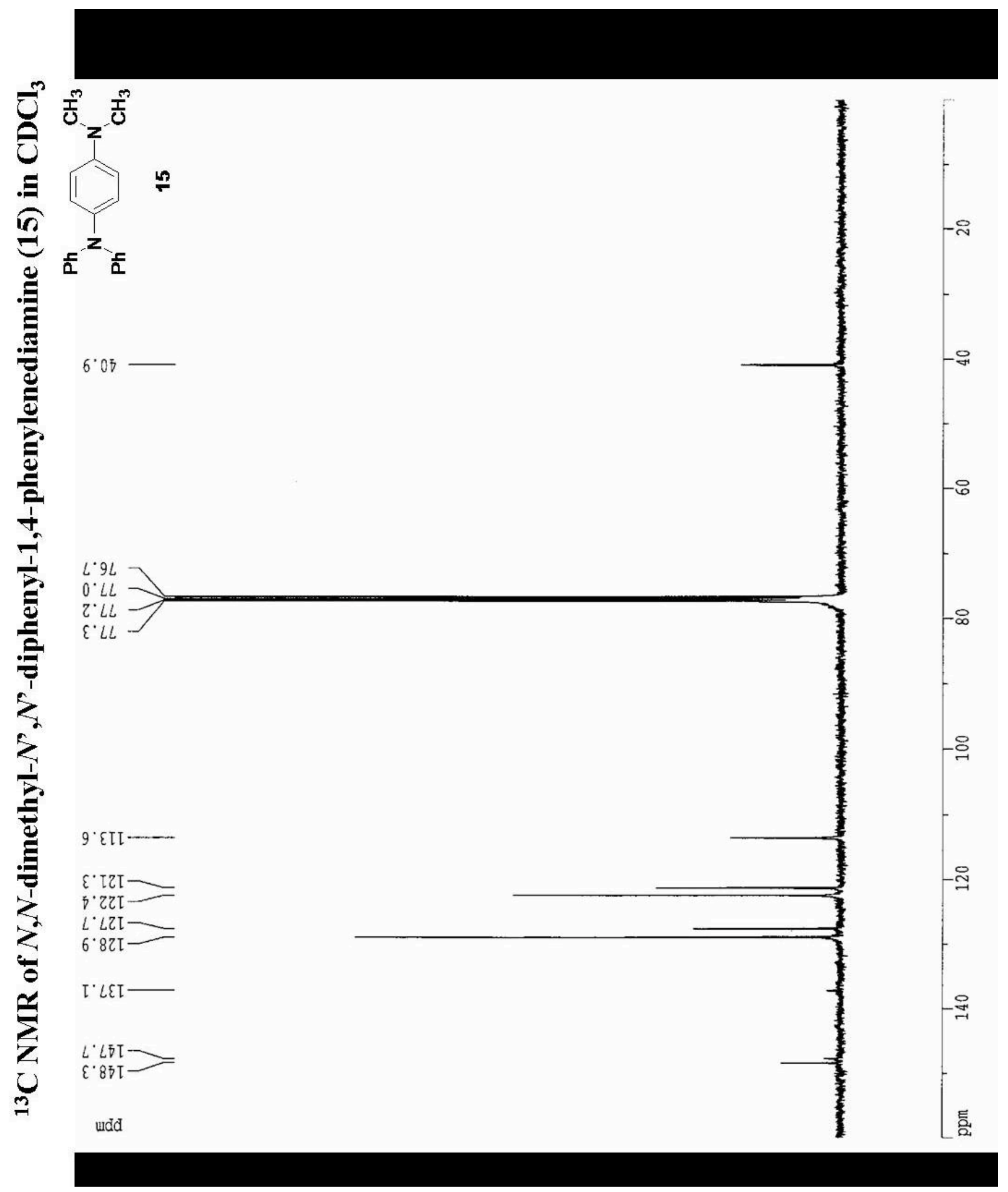




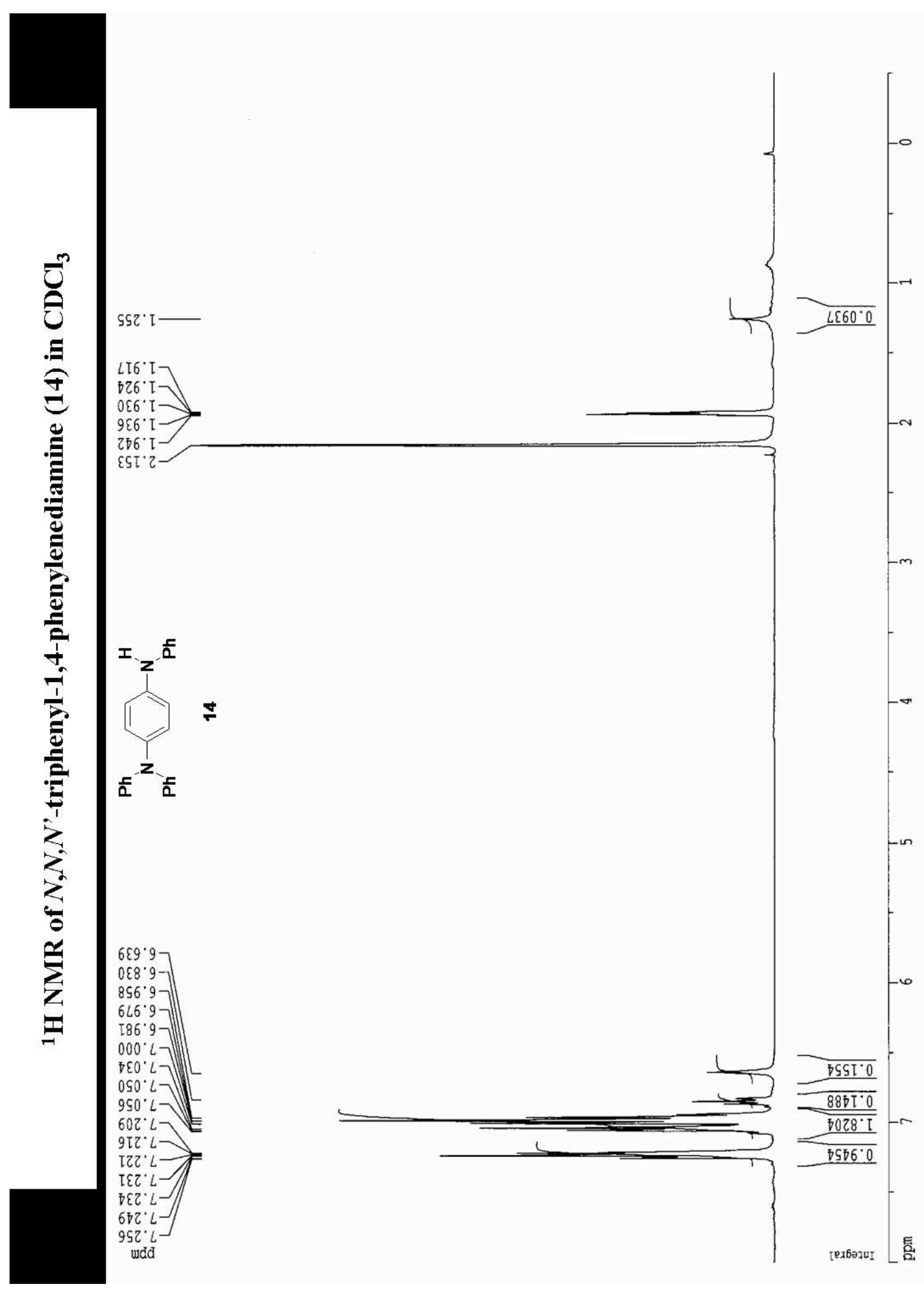




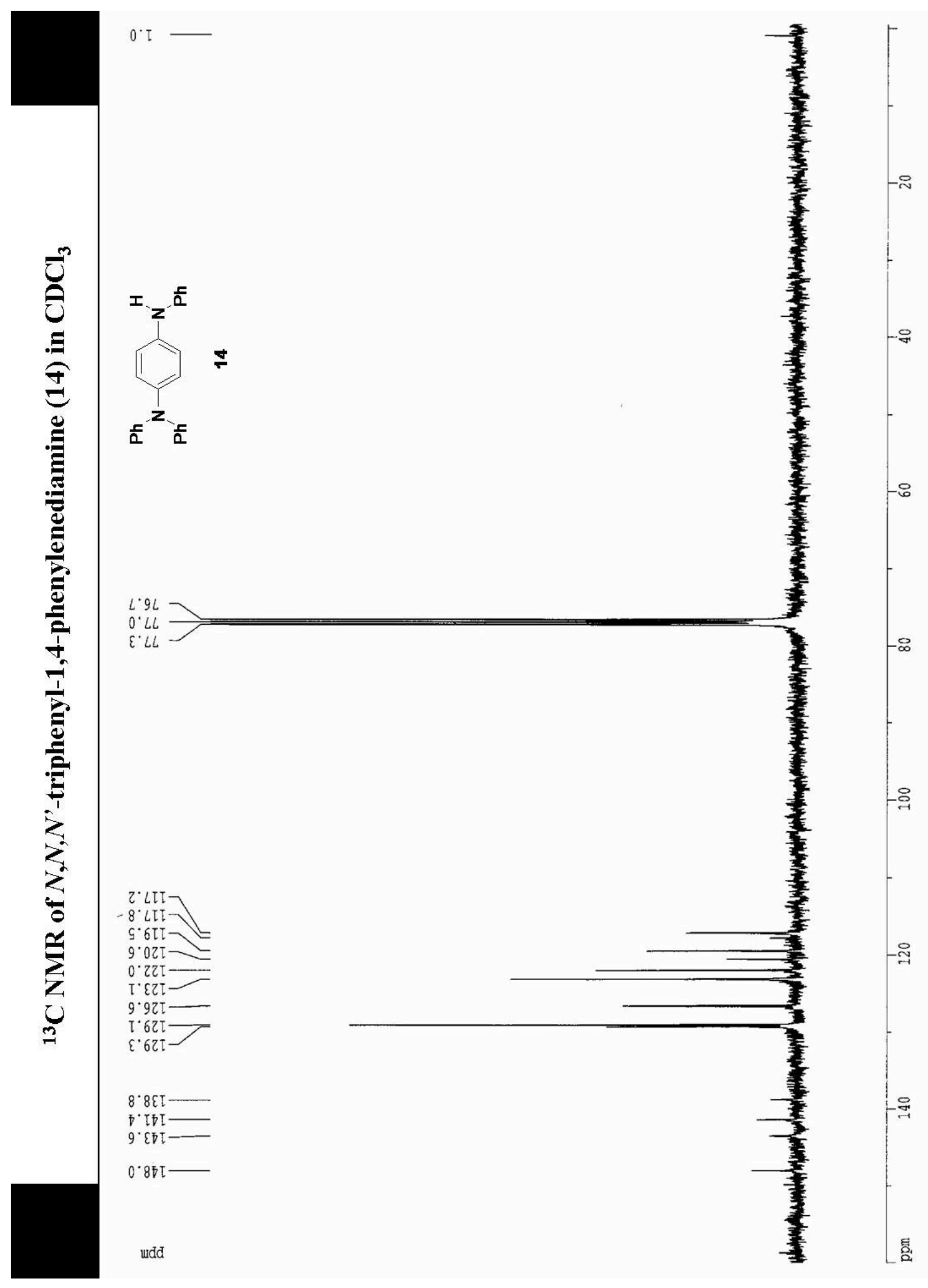




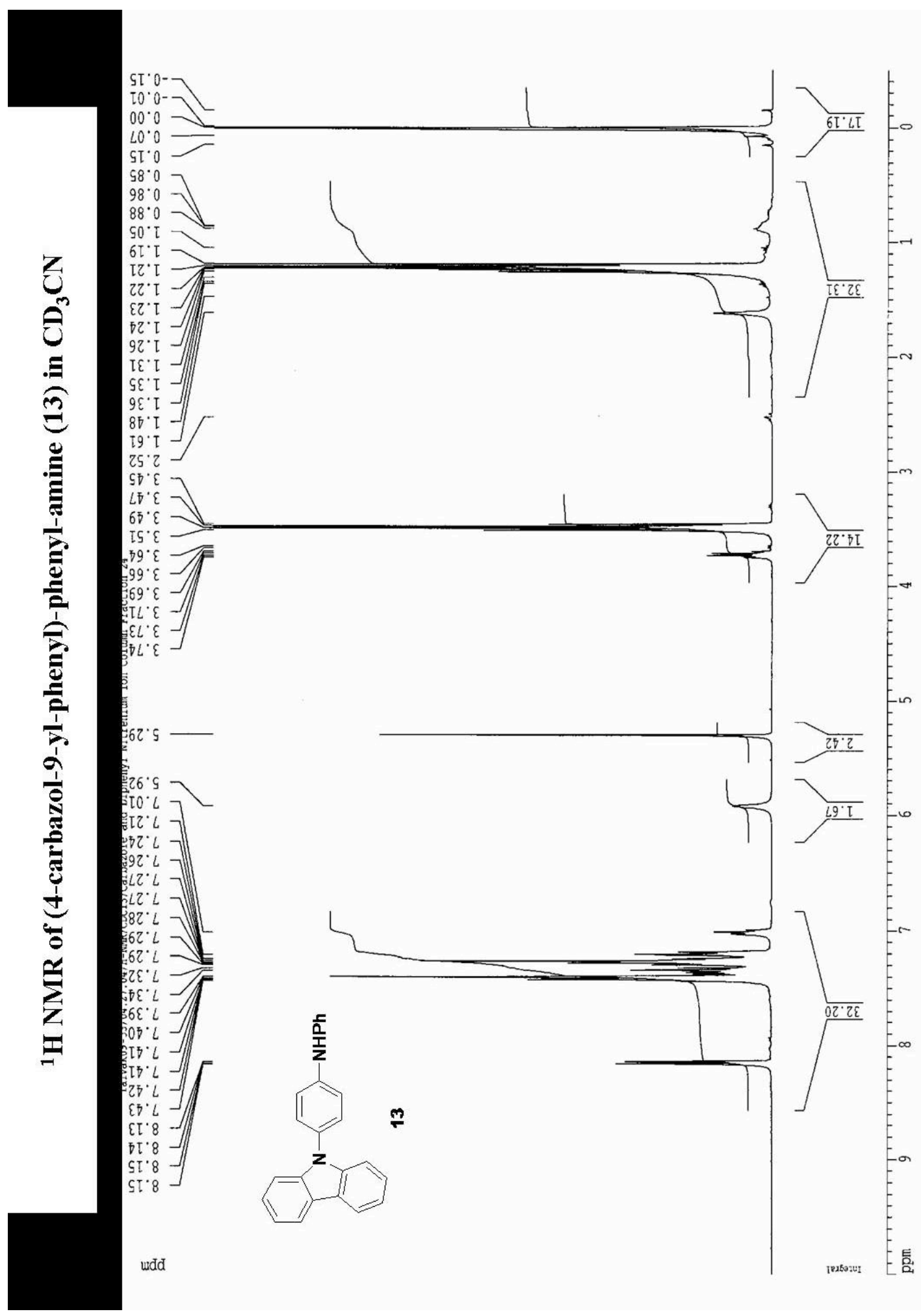




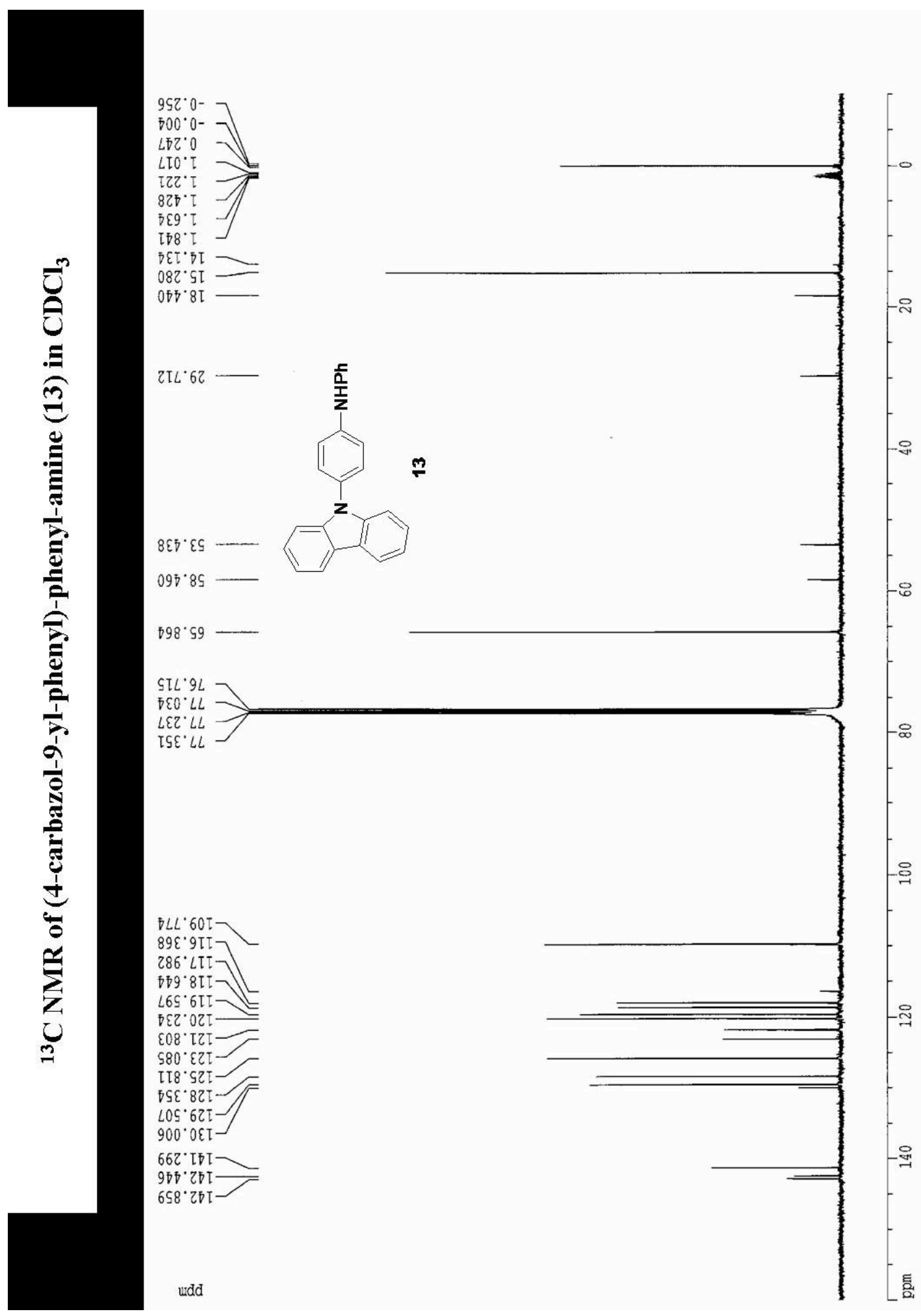




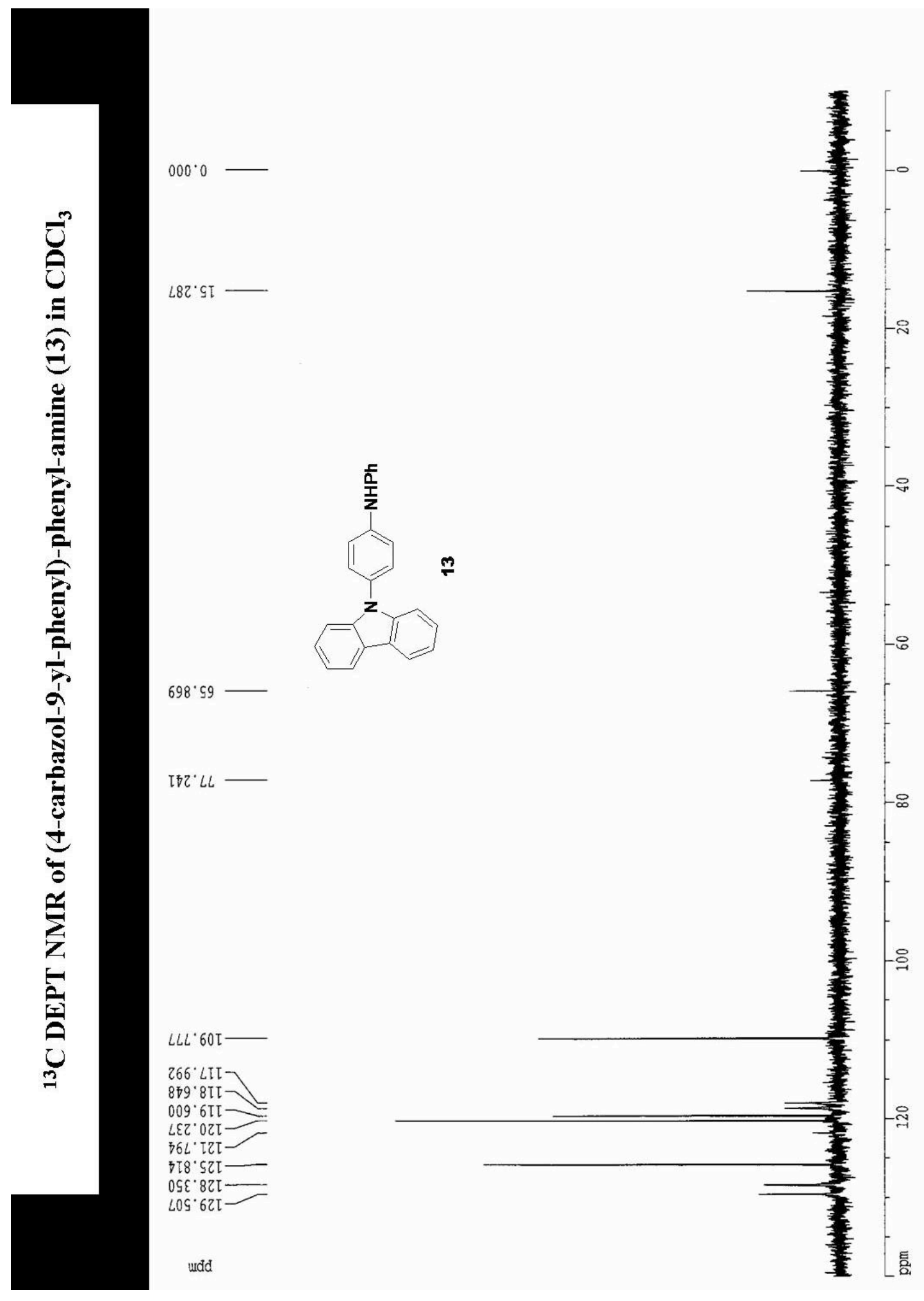




\section{Transition State 12}

Zero-point correction $=$

Thermal correction to Energy=

Thermal correction to Enthalpy=

Thermal correction to Gibbs Free Energy=

Sum of electronic and zero-point Energies=

Sum of electronic and thermal Energies $=$

Sum of electronic and thermal Enthalpies=

Sum of electronic and thermal Free Energies=
.186023 (Hartree/Particle)

.194940

.195884

.152347

$-517.568283$

$-517.559365$

$-517.558421$

$-517.601958$

\begin{tabular}{|c|c|c|c|c|c|}
\hline \multirow{2}{*}{$\begin{array}{l}\text { Center } \\
\text { Number }\end{array}$} & \multirow{2}{*}{\multicolumn{2}{|c|}{$\begin{array}{l}\text { Atomic } \\
\text { Number }\end{array}$}} & Atomic & \multicolumn{2}{|c|}{ Coordinates (Angstroms } \\
\hline & & & Type & $X$ & $\mathrm{Z}$ \\
\hline 1 & 5 & 0 & .000000 & .000000 & 000000 \\
\hline 2 & 6 & 0 & .000000 & .000000 & 1.432310 \\
\hline 3 & 6 & 0 & 1.168152 & .000000 & 2.156125 \\
\hline 4 & 6 & 0 & 2.399240 & -.068661 & 1.451135 \\
\hline 5 & 6 & 0 & 2.418256 & .051069 & -.020251 \\
\hline 6 & 6 & 0 & 1.168880 & .036545 & -.718635 \\
\hline 7 & 1 & 0 & -.952618 & .037398 & -.518977 \\
\hline 8 & 1 & 0 & -.952542 & -.021447 & 1.952256 \\
\hline 9 & 1 & 0 & 1.180547 & -.061538 & 3.238510 \\
\hline 10 & 1 & & 1.153943 & .148562 & -1.797717 \\
\hline 11 & 6 & 6 & 4.367269 & -.955744 & 1.143004 \\
\hline 12 & 6 & 6 & 5.782620 & -.896140 & 1.245632 \\
\hline 13 & 6 & 6 & 6.531287 & -1.188144 & .130859 \\
\hline 14 & 6 & 6 & 5.924592 & -1.610467 & $7 \quad-1.095956$ \\
\hline 15 & 6 & 6 & 4.566735 & -1.778559 & $9-1.205601$ \\
\hline 16 & 6 & 6 & 3.734899 & -1.502432 & $2 \quad-.073924$ \\
\hline 17 & 1 & 1 & 6.225758 & -.517503 & 2.159832 \\
\hline 18 & 1 & 1 & 7.610569 & -1.078473 & .166408 \\
\hline 19 & 1 & 1 & 6.567836 & $5-1.863221$ & $1-1.932956$ \\
\hline 20 & 1 & 1 & 4.130689 & -2.204507 & $7 \quad-2.103165$ \\
\hline 21 & 7 & 7 & 3.553315 & -.393823 & 2.042440 \\
\hline 22 & 1 & 1 & 2.904995 & -2.187661 & .083499 \\
\hline 23 & 1 & 1 & 3.188540 & 694795 & -.438986 \\
\hline
\end{tabular}

Intermediate $\mathbf{1 0}$

Zero-point correction=

Thermal correction to Energy=

Thermal correction to Enthalpy=

Thermal correction to Gibbs Free Energy=

Sum of electronic and zero-point Energies=
.186023 (Hartree/Particle)

.194940

.195884

.152347

$-517.568283$ 
Sum of electronic and thermal Energies=

$-517.559365$

Sum of electronic and thermal Enthalpies=

$-517.558421$

Sum of electronic and thermal Free Energies=

$-517.601958$

Center Atomic Atomic Coordinates (Angstroms)

$\begin{array}{cccccc}\text { Number } & \text { Number } & \text { Type } & X & Y & Z \\ - & \text { X } & & \\ 1 & 6 & 0 & 3.063346 & -1.090241 & -.081760 \\ 2 & 6 & 0 & 3.382919 & .318052 & -.097027 \\ 3 & 6 & 0 & 2.421778 & 1.302188 & .034736 \\ 4 & 6 & 0 & 1.077571 & .902958 & .173439 \\ 5 & 6 & 0 & .705945 & -.548459 & .398470 \\ 6 & 6 & 0 & 1.789796 & -1.527041 & .128906 \\ 7 & 1 & 0 & 3.869435 & -1.802324 & -.216365 \\ 8 & 1 & 0 & 4.416631 & .605697 & -.258939 \\ 9 & 1 & 0 & 2.658786 & 2.354488 & -.062709 \\ 10 & 1 & 0 & 1.570051 & -2.587223 & .207239 \\ 11 & 6 & 0 & -1.077573 & .902962 & -.173453 \\ 12 & 6 & 0 & -2.421776 & 1.302189 & -.034738 \\ 13 & 6 & 0 & -3.382919 & .318050 & .097022 \\ 14 & 6 & 0 & -3.063347 & -1.090241 & .081756 \\ 15 & 6 & 0 & -1.789795 & -1.527041 & -.128903 \\ 16 & 6 & 0 & -.705945 & -.548458 & -.398465 \\ 17 & 1 & 0 & -2.658787 & 2.354487 & .062717 \\ 18 & 1 & 0 & -4.416630 & .605697 & .258938 \\ 19 & 1 & 0 & -3.869435 & -1.802326 & .216362 \\ 20 & 1 & 0 & -1.570050 & -2.587223 & -.207229 \\ 21 & 7 & 0 & .000000 & 1.703135 & .000011 \\ 22 & 1 & 0 & -.409797 & -.676361 & -1.454569 \\ 23 & 1 & 0 & .409800 & -.676355 & 1.454577\end{array}$

Intermediate $\mathbf{1 1}$

$E=-517.2760$ Hartrees

\begin{tabular}{|c|c|c|c|c|}
\hline \multirow{2}{*}{$\begin{array}{l}\text { Center } \\
\text { Number }\end{array}$} & \multirow{2}{*}{$\begin{array}{l}\text { Atomic } \\
\text { Number }\end{array}$} & \multirow{2}{*}{$\begin{array}{r}\text { Atomic } \\
\text { Type }\end{array}$} & \multicolumn{2}{|c|}{ Coordinates (Angstroms } \\
\hline & & & $\mathrm{X}$ & $\mathrm{Z}$ \\
\hline 1 & 6 & -2.970878 & 1.126511 & -.349273 \\
\hline 2 & 6 & -3.385578 & -.263179 & -.165198 \\
\hline 3 & 6 & -2.490021 & -1.257785 & 100006 \\
\hline 4 & 6 & -1.097954 & -.907618 & 212450 \\
\hline
\end{tabular}




$\begin{array}{cccccc}5 & 6 & 0 & -.717823 & .551899 & .463537 \\ 6 & 6 & 0 & -1.698004 & 1.529747 & -.125205 \\ 7 & 1 & 0 & -3.715939 & 1.836322 & -.695953 \\ 8 & 1 & 0 & -4.433778 & -.504499 & -.313159 \\ 9 & 1 & 0 & -2.776039 & -2.303510 & .118109 \\ 10 & 1 & 0 & -1.392868 & 2.561906 & -.271054 \\ 11 & 6 & 0 & 1.092484 & -.866726 & .003047 \\ 12 & 6 & 0 & 2.409848 & -1.278881 & -.195211 \\ 13 & 6 & 0 & 3.408954 & -.296885 & -.231287 \\ 14 & 6 & 0 & 3.093416 & 1.060386 & -.062069 \\ 15 & 6 & 0 & 1.766214 & 1.470422 & .157527 \\ 16 & 6 & 0 & .768790 & .502996 & .177312 \\ 17 & 1 & 0 & 2.638528 & -2.331072 & -.320166 \\ 18 & 1 & 0 & 4.441838 & -.588774 & -.392754 \\ 19 & 1 & 0 & 3.883512 & 1.803485 & -.102421 \\ 20 & 1 & 0 & 1.537587 & 2.522916 & .298331 \\ 21 & 7 & 0 & -.065717 & -1.701892 & .028496 \\ 22 & 1 & 0 & -.799501 & .691146 & 1.565781\end{array}$

Diphenylnitrenium ion. 1

Zero-point correction $=$

Thermal correction to Energy=

Thermal correction to Enthalpy=

Thermal correction to Gibbs Free Energy=

Sum of electronic and zero-point Energies=

Sum of electronic and thermal Energies $=$

Sum of electronic and thermal Enthalpies=

Sum of electronic and thermal Free Energies=
.186335 (Hartree/Particle) .196121 .197065

.151234

$-517.594803$

$-517.585017$

$-517.584073$

$-517.629904$

Center Atomic Atomic Coordinates (Angstroms)

Number Number Type X $\quad$ Y $\quad$ Z

$\begin{array}{cccccc}1 & 6 & 0 & -.161912 & 1.208029 & .414946 \\ 2 & 6 & 0 & -.605677 & 1.447016 & -.932004 \\ 3 & 6 & 0 & -.725550 & 2.746134 & -1.391444 \\ 4 & 6 & 0 & -.403534 & 3.832535 & -.546393 \\ 5 & 6 & 0 & .000000 & 3.622353 & .785588 \\ 6 & 6 & 0 & .085827 & 2.329682 & 1.276642 \\ 7 & 1 & 0 & -.923490 & .618856 & -1.553477 \\ 8 & 1 & 0 & -1.089452 & 2.937643 & -2.394143 \\ 9 & 1 & 0 & -.500975 & 4.845255 & -.923046 \\ 10 & 1 & 0 & .219582 & 4.467974 & 1.426048 \\ 11 & 1 & 0 & .373520 & 2.119350 & 2.299823 \\ 12 & 7 & 0 & .000000 & .000000 & 1.000229 \\ 13 & 6 & 0 & .161912 & -1.208029 & .414946\end{array}$




$\begin{array}{llllll}14 & 6 & 0 & .605677 & -1.447016 & -.932004 \\ 15 & 6 & 0 & -.085827 & -2.329682 & 1.276642 \\ 16 & 6 & 0 & .725550 & -2.746134 & -1.391444 \\ 17 & 1 & 0 & .923490 & -.618856 & -1.553477 \\ 18 & 6 & 0 & .000000 & -3.622353 & .785588 \\ 19 & 1 & 0 & -.373520 & -2.119350 & 2.299823 \\ 20 & 6 & 0 & .403534 & -3.832535 & -.546393 \\ 21 & 1 & 0 & 1.089452 & -2.937643 & -2.394143 \\ 22 & 1 & 0 & -.219582 & -4.467974 & 1.426048 \\ 23 & 1 & 0 & .500975 & -4.845255 & -.923046\end{array}$

